\title{
GROUPS WITH ISOMORPHIC PROPER SUBGROUPS
}

\section{ROSS A. BEAUMONT}

1. Introduction. Since isomorphic groups have orders which are the same cardinal number, no finite group has an isomorphic proper subgroup. Moreover if there exists an isomorphism $f_{1}$ of a group $G$ such that $G^{f_{1}}$ is a proper subgroup of $G$, then $f_{1}$ induces an isomorphism $f_{2}$ of $G^{f_{1}}$ upon a proper subgroup $\left(G^{f_{1}}\right)^{f_{2}}=G^{f_{1 / 2}}$ of $G^{f_{1}}$. An infinite chain of isomorphic subgroups is generated such that

$$
G>G^{f_{1}}>G^{f_{1} f_{2}}>\cdots>G^{f_{1} f_{2}} \cdots f_{n}>\cdots>1 .
$$

Thus any group $G$ with an isomorphic proper subgroup does not satisfy the descending chain condition. Therefore a survey of groups with isomorphic proper subgroups is concerned only with infinite groups which do not satisfy the descending chain condition. Among these groups, it is obvious that infinite cyclic groups have isomorphic proper subgroups.

In a recent paper ${ }^{1}$ Baer has given a criterion for groups which do not have isomorphic proper subgroups. This criterion has not been used in the present note since the structure of the groups considered allows simpler proofs. Because of its interest in this problem, however, it is repeated here.

CRITERION. The group $G$ does not have an isomorphic proper subgroup if there exists a well ordered ascending chain of subgroups $N(v)$ of $G$ with the following properties.

(i) $N(v)^{f} \leqq N(v)$ for every isomorphism $f$ of $G$ into itself.

(ii) $N(0)=1$ and $N(t)=G$ for some ordinal $t$.

(iii) $N(v+1) / N(v)$ does not have an isomorphic proper subgroup.

(iv) If $v$ is a limit ordinal, then every element of $N(v)$ is contained in some $N(u)$ for $u<v$.

We shall call a group $G$ completely reducible if it is the direct product of groups of rank 1 . The theorems of this note give a complete survey of those completely reducible groups which have isomorphic proper subgroups.

Definition 1. A group $G$ is an I-group if there exists a proper sub-

Presented to the Society, November 25, 1944; received by the editors September, 1944, and, in revised form, November 29, 1944.

1 R. Baer, Groups without proper isomorphic quotient groups, Bull. Amer. Math. Soc. vol. 50 (1944) pp. 267-277. 
group $H$ of $G$ such that $G$ and $H$ are isomorphic.

LEMMA 1. If a group $G$ has a direct factor $S$ which is an I-group, then $G$ is an $I$-group.

Proof. $G=S \times T$ for some subgroup $T$ of $G$ and there exists a proper subgroup $S^{\prime}$ of $S$ such that $S$ and $S^{\prime}$ are isomorphic. The product group $H=S^{\prime} T=S^{\prime} \times T$ is a proper subgroup of $G$ and is isomorphic to $G$ since the corresponding direct factors $S$ and $S^{\prime}, T$ and $T$, of $G$ and $H$ are isomorphic. Hence $G$ is an $I$-group.

LEMMA 2. An infinite direct power group, that is, a group $G$ which is the direct product of infinitely many groups which are isomorphic to each other, is an I-group.

Proof. There is a (1-1) correspondence between the direct factors of $G$ and the direct factors of any proper subgroup $H$ which is the direct product of almost all of the direct factors of $G$, and the corresponding direct factors are isomorphic.

2. Abelian groups all of whose elements are of finite order. The following result proves that the discussion of abelian groups all of whose elements are of finite order may be limited to primary abelian groups without loss in generality.

LEMma 3. If $A$ is an abelian group such that all the elements of $A$ are of finite order, then $A$ is an I-group if, and only if, one of the primary components $A(p)$ of $A$ is an $I$-group.

Proof. $A=\prod_{q} A(q)$ where the $A(q)$ are primary abelian groups. If $A(p)$ is an $I$-group for some $p$, it follows from Lemma 1 that $A$ is an $I$-group. Conversely, if $A$ is an $I$-group, then there exists a proper subgroup $A^{\prime}$ of $A$ such that $A$ and $A^{\prime}$ are isomorphic. Since the orders of elements are preserved by isomorphism, the isomorphism of $A$ on $A^{\prime}$ induces an isomorphism of $A(q)$ on $A^{\prime}(q)$ for all $q$, where $A^{\prime}(q)$ is the primary $q$-component of $A^{\prime}$. Now $A^{\prime}(q) \leqq A(q)$ for all $q$ since $A^{\prime}<A$, and it follows from $A^{\prime}=\prod_{q} A^{\prime}(q)$ that for some $p, A^{\prime}(p)$ $<A(p)$, for otherwise $A=A^{\prime}$. Thus $A(p)$ is an $I$-group.

Definition 2. $A$ group $G$ is said to be of finite rank $r(G)$ if there exists an integer $r(G)$ with the property that $r(G)$ is the smallest number such that every finite subset of elements of $G$ which does not consist of the unity element alone is contained in a subgroup of $G$ which is generated by $r(G)$ elements. The rank of the unit group $\{1\}$ is defined to be 0 . If no such integer exists, then $G$ is said to be of infinite rank and we write $r(G)=\infty$. 
It is known ${ }^{2}$ that the only primary abelian groups of rank 1 are cyclic groups and groups of type $p^{\infty} . G$ is a group of type $p^{\infty}$ if $G$ is generated by an infinite sequence $a(i)$ of elements where $a(0)$ is of order $p$ and $a(i+1)^{p}=a(i)$ for every $i$.

TheOREM 1. If $A$ is a primary abelian group of finite rank $r$, then $A$ is not an I-group.

Proof. It follows from the hypothesis and known results ${ }^{3}$ that $A$ is the direct product of groups of rank 1 except in the trivial case where $r=0$ and $A=\{1\}$. Assume that $A$ is an $I$-group. Denote by $A\left(p^{k}\right)$ the subgroup of $A$ consisting of all those elements of $A$ whose orders divide $p^{k}$. The definition of a group of type $p^{\infty}$ implies that $A\left(p^{k}\right)$ is a finite group. The isomorphism of $A$ on a proper subgroup $A^{\prime}$ induces an isomorphism of $A\left(p^{k}\right)$ on a subgroup $A^{\prime}\left(p^{k}\right)$ of $A^{\prime}$. Since orders are preserved and since $A^{\prime}<A, A^{\prime}\left(p^{k}\right) \leqq A\left(p^{k}\right)$. Since no finite group is an $I$-group, $A^{\prime}\left(p^{k}\right)=A\left(p^{k}\right)$. If $a$ is an element of $A, a$ is contained in $A\left(p^{j}\right)$ for some integer $j$. By the above $A\left(p^{j}\right)=A^{\prime}\left(p^{j}\right)$ $<A^{\prime}$ so that $a$ is contained in $A^{\prime}$. Thus $A \leqq A^{\prime}$ which contradicts $A^{\prime}<A$ and the assumption that $A$ is an $I$-group.

REMARK. Note that the subgroups $A\left(p^{0}\right), A\left(p^{1}\right), \cdots, A\left(p^{k}\right), \cdots$ form an ascending chain of subgroups of $A$ which have the properties of Baer's criterion.

THEOREM 2. If $A$ is a primary abelian group of infinite rank and if $A$ is the direct product of groups of rank 1 , then $A$ is an I-group.

Proof. Case I. The orders of the elements of $A$ are bounded.

$A$ is the direct product of infinitely many cyclic groups of order dividing $p^{k}$ for some integer $k$. In this direct factorization there must be infinitely many cyclic groups of order $p^{i}$ for some $i$ where $k \geqq i>0$. The direct product of these groups is an infinite direct power group, and hence by Lemmas 2 and $1, A$ is an $I$-group.

Case II. The orders of the elements of $A$ are not bounded.

If there are infinitely many direct factors which are groups of type $p^{\infty}$, then the direct product of these groups is an infinite direct power group, and $A$ is an $I$-group as in Case $I$.

If there are only a finite number of direct factors of type $p^{\infty}$, then since $A$ is of infinite rank, there are infinitely many cyclic groups in the direct factorization. If there are infinitely many cyclic direct factors of order $p^{i}$ for some integer $i$, then the proof that $A$ is an $I$-group

${ }^{2} \mathrm{H}$. Prüfer, Untersuchungen uber die Zerlegbarkeit der abzahlbaren primaren Abelschen Gruppen, Math. Zeit. vol. 17 (1923) pp. 35-61.

${ }^{3}$ H. Prüfer, ibid. 
is that of Case I. The alternative is that there be only a finite number of cyclic direct factors of $A$ of order $p^{i}$ for all $i$, so that in the direct factorization of $A$ there are cyclic groups of arbitrarily high order. (Otherwise, $A$ would be of finite rank.) Then

$$
A=\prod_{i=1}^{n} G_{i}(\infty) \times \prod_{i=1}^{n_{1}} G_{i}\left(j_{1}\right) \times \prod_{i=1}^{n_{2}} G_{i}\left(j_{2}\right) \times \cdots \times \prod_{i=1}^{n_{k}} G_{i}\left(j_{k}\right) \times \cdots
$$

where $G_{1}(\infty)=\{1\} ; G_{i}(\infty), i \neq 1$, is of type $p^{\infty} ; G_{i}\left(j_{k}\right)$ is cyclic of order $q_{k}=p^{i_{k}}$, and $j_{1}<j_{2}<\cdots<j_{k}<\cdots$. Let

$$
\begin{aligned}
A^{\prime}=\prod_{i=1}^{n} G_{i}(\infty) & \times \prod_{i=1}^{n_{1}-1} G_{i}\left(j_{1}\right) \times\left(G_{n_{2}}\left(j_{2}\right)\right)^{q_{2} / q_{1}} \times \prod_{i=1}^{n_{2}-1} G_{i}\left(j_{2}\right) \\
& \times\left(G_{n_{3}}\left(j_{3}\right)\right)^{q_{8} / q_{2}} \times \cdots
\end{aligned}
$$

where if $n_{k}-1=0$,

is replaced by $\{1\}$.

$$
\prod_{i=1}^{n_{k}-1} G_{i}\left(j_{k}\right)
$$

By construction, $A^{\prime}$ is a proper subgroup of $A$ and there is a (1-1) correspondence between the direct factors of $A$ and those of $A^{\prime}$. Since these corresponding direct factors are isomorphic, $A$ and $A^{\prime}$ are isomorphic and $A$ is an $I$-group.

3. Abelian groups all of whose elements are of infinite order. In this section, the abelian groups considered will be written additively.

THeOREM 3. If $G$ is an abelian group such that all the elements of $G$ except the identity are of infinite order, and if $G$ does not admit the field of rational numbers, $R$, as a field of operators, then $G$ is an I-group.

Proof. If $\beta G=G$ for every integer $\beta \neq 0$, then for every integer $\alpha$ and every $g$ in $G, \alpha g=\beta g^{\prime}$ for some $g^{\prime}$ in $G$. Thus the equation $\alpha g=\beta x$ has a solution $g^{\prime}$ in $G$ for every pair of integers $\alpha$ and $\beta \neq 0$ and for every $g$ in $G$. This is just the statement that $G$ admits $R$, which contradicts the hypothesis. Hence there exists an integer $\beta \neq 0$ such that $\beta G$ is a proper subgroup of $G$. Moreover, the homomorphism $g \rightarrow \beta g$ is an isomorphism of $G$ on $\beta G$, for if $\beta g=\beta f, \beta(g-f)=0$; and since $\beta \neq 0$, this implies that $g-f$ has finite order. By the hypothesis on $G$, $g-f=0$ and $g=f$.

4 If $G$ admits $R$, the equation has a unique solution, but the existence of a solution implies its uniqueness. For a discussion of the properties of rational numbers as operators of the abelian group, see R. Baer, Abelian groups without elements of finite order, Duke Math. J. vol. 3 (1937) p. 70. 
COROLlaRY. Every proper subgroup, $G \neq 0$, of the additive group of all rational numbers, $R$, is an $I$-group.

Proof. Assume that $G$ admits $R$ as a field of operators. Let $s / t$ be an element of $G$ where $s$ and $t$ are nonzero integers. Then $r(s / t)=g$ in $G$ for every $r$ in $R$, and hence $(t / s) g$ is in $G$. But $(t / s) g=(t / s)[r(s / t)]=r$, so that $r$ is in $G$ for every $r$ in $R$. This contradicts $G<R$ and with Theorem 3 completes the proof of the corollary.

Theorem 4. If $G$ admits $R$ as a field of operators, then $G$ is an $I$-group if, and only if, $G$ is infinite-dimensional over $R$.

Proof. If $G$ is infinite-dimensional over the field $R, G$ possesses an infinite basis, ${ }^{5} B$, with respect to $R$. Let $G^{\prime}$ be the subgroup of $G$ with basis $B^{\prime}$, where $B^{\prime}$ contains almost all of the elements of $B$. The (1-1) correspondence between $B$ and $B^{\prime}$ may be extended to an isomorphism of $G$ upon its proper subgroup $G^{\prime}$ in the usual way.

Conversely, if $G$ is an $I$-group which admits $R$, then $G$ contains an isomorphic proper subgroup $G^{\prime}$ which admits $R$. For if $g^{\prime}$ is in $G^{\prime}$ and $s$ and $t \neq 0$ are integers, there exists an element $h$ of $G$ such that $s g=t h$, where $g$ is the correspondent of $g^{\prime}$ under the isomorphism of $G$ upon $G^{\prime}$. We have

$$
s g^{\prime}=(s g)^{\prime}=(t h)^{\prime}=t h^{\prime}
$$

where $h^{\prime}$ is in $G^{\prime}$, and this is the statement that $G^{\prime}$ admits $R$. It follows from the above equation that the isomorphism of $G$ upon $G^{\prime}$ is an operator-isomorphism. If the dimension of $G$ over $R$ is finite, $G^{\prime}$ would have the same finite dimension over $R$, and this would imply $G=G^{\prime}$ which contradicts the hypothesis. Hence $G$ is of infinite dimension over $R$.

CoRollary 1. If $G$ is the direct sum of $\omega$ groups each isomorphic to $R$, then $G$ is an I-group if, and only if, $\omega$ is infinite.

PRoof. $G$ admits $R$ as a field of operators and $G$ is infinite-dimensional over $R$ if, and only if, $\omega$ is infinite.

CoROLlaRy 2. The additive group of all rational numbers, $R$, is not an I-group.

PRoof. $R$ satisfies the hypothesis of Corollary 1 with $\omega=1$.

It is interesting to note that the hypothesis of Theorem 4, that an abelian group $G$ admits the field $R$ of rational numbers as an operator domain, implies that every element of $G$ except the identity is of in-

${ }^{5}$ H. Zassenhaus, Lehrbuch der Gruppenthorie, p. 66, Satz 15. 
finite order. For if $n g=0$ with $g$ in $G$ and $n$ a nonzero integer, $(1 / n)(n g)=g=0$.

The results of this section may be generalized in the following way. ${ }^{\circ}$ Let $G$ be an abelian group admitting an integral domain $D$ of operators. Let $F$ be the quotient field of $D$.

Definition 3. $G$ is an I-group with respect to $D$, or more simply, a $D$-group, if $G$ contains an operator-isomorphic proper subgroup.

The following theorems whose proofs are simplified versions of the proofs of Theorem 3 and Theorem 4 may be stated.

TheOREM 5. If $G$ is an abelian group admitting $D$ as a domain of operators, such that $\alpha g=0$ for $\alpha$ in $D$ and $g$ in $G$ implies that either $\alpha=0$ or $g=0$, and if $G$ does not admit $F$ as a field of operators, then $G$ is a D-group.

THEOREM 6. If $G$ admits $F$ as a field of operators, then $G$ is a $D$-group if, and only if, $G$ is infinite-dimensional over $D$.

4. Summary. If $H$ is a group of rank 1 , then $H$ is an unmixed group, that is, either every element of $H$ is of finite order or every element of $H$ except the identity is of infinite order. If $H$ does not contain elements of infinite order, the primary components of $H$ are either finite cyclic groups or groups of type $p^{\infty}$. If $H$ does contain elements of infinite order, $H$ is essentially a subgroup of the additive group of all rational numbers, ${ }^{7} R$. Hence if a group $G$ is completely reducible it is the direct sum of groups of the three types mentioned above. Let $F(G)$ be the direct sum of those direct summands of $G$ which do not contain elements of infinite order. Then $F(G)$ is the subgroup of all of the elements of finite order in $G$, and $G=F(G)+I(G)$ where $I(G)$ is the direct sum of rational groups.

THEOREM 7. If $G$ is a completely reducible group, then $G$ is an I-group if, and only if, either $F(G)$ or $I(G)$ is an I-group.

PRoof. If either $F(G)$ or $I(G)$ is an $I$-group, then $G$ is an $I$-group by Lemma 1 . If $G$ contains an isomorphic proper subgroup $G^{\prime}$, then $G^{\prime}=F^{\prime}(G)+I^{\prime}(G)$ where $F^{\prime}(G)$ is the map of $F(G)$ and $I^{\prime}(G)$ is the map of $I(G)$ under the isomorphism. But $F^{\prime}(G) \leqq F(G)$ since orders are preserved. If $F^{\prime}(G)<F(G)$, the theorem is proved; therefore, let us assume that $F^{\prime}(G)=F(G) . I(G)$ is isomorphic to the differ-

- The author wishes to thank the referee for suggesting these generalizations with their proofs, which in turn suggested a revision of this section.

${ }^{7}$ See the reference of footnote 4. 
ence group $G-F(G)$ which contains properly the difference group $G^{\prime}-F^{\prime}(G)$ since $F^{\prime}(G)=F(G)$. Now $I^{\prime}(G)$ is isomorphic to $G^{\prime}-F^{\prime}(G)$ and to $I(G)$. Hence $G-F(G)$ is an $I$-group and it follows that $I(G)$ which is isomorphic to $G-F(G)$ is also an $I$-group.

It follows from Theorem 7 that the theorems and corollaries of $\$ \$ 2$ and 3 survey completely all completely reducible groups, $G$, which are $I$-groups.

UNIVERSITY OF WASHINGTON

\section{AN EXISTENCE THEOREM FOR LATIN SQUARES}

\section{MARSHALL HALL}

1. Introduction. A latin square may be interpreted as a representation of a 3-web or as the multiplication table of a quasi-group. Hence the following theorem has application both in the theory of projective planes and in the theory of quasi-groups. It is derived from a very interesting result of $\mathrm{P}$. Hall.

2. The existence theorem. Is there any combinatorial restriction which prevents us from constructing a latin square by adding a row at a time? The following theorem shows that such a procedure is permissible.

THEOREM. Given a rectangle of $n-r$ rows and $n$ columns such that each of the numbers $1,2, \cdots, n$ occurs once in every row and no number occurs twice in any column, then there exist $r$ rows which may be added to the given rectangle to form a latin square.

Proof. Let $C_{i}, i=1,2, \cdots, n$ be the subset of the numbers $1,2, \cdots, n$ which do not occur in the $i$ th column of the given rectangle. Then each $C_{i}$ contains $r$ numbers and each number occurs $r$ times in all the $C$ 's. For there are $n-r$ numbers in the $i$ th column and each number has appeared in $n-r$ columns. It will be shown that the subsets satisfy the requirements of P. Hall's theorem $:^{1}$

In order that a complete system of distinct representatives of subsets

Received by the editors January 19, 1945.

Opinions and assertions in this paper are those of the author and are not to be construed as official or as reflecting the views of the United States Navy Department or the Naval Service at large. 30.

${ }^{1}$ P. Hall, On representatives of subsets, J. London Math. Soc. vol. 10 (1935) pp. 26- 